\title{
Representaciones sociales y prácticas pedagógicas en educación ambiental'
}

\author{
Raúl Calixto-Flores ${ }^{2}$ \\ ORCID: 0000-0001-7940-3299
}

\section{Resumen}

Las representaciones sociales constituyen un conocimiento práctico con el cual se da significado al medio ambiente y sentido a distintas prácticas. Los conocimientos de las representaciones sociales de la educación ambiental constituyen la base para la comprensión de las prácticas de los educadores ambientales. La investigación realizada tiene el objetivo de describir las representaciones de educación ambiental y el sentido de las prácticas pedagógicas de una muestra de educadores ambientales. Es un estudio descriptivo, de carácter interpretativo, orientado por el enfoque procesual de las representaciones sociales. Entre los resultados obtenidos, se observa que los educadores ambientales les confieren a las prácticas pedagógicas en educación ambiental un sentido hacia la conformación de estilos de vida congruentes con actitudes y valores de cuidado y respeto al medio ambiente. En la investigación se observa la existencia de representaciones sociales sobre educación ambiental y algunas de las implicaciones que tienen en sus prácticas pedagógicas. Estos resultados confirman porque las representaciones sociales constituyen una teoría que cada vez más se utiliza para comprender los conocimientos de sentido común que poseen los diversos actores educativos, los cuales pueden tomar una postura en torno a la práctica pedagógica. De igual forma, contribuye a interpretar las relaciones entre las representaciones y prácticas pedagógicas en educación ambiental. Predominan representaciones sociales antropocéntricas, por lo que en los procesos de formación y/o actualización de los educadores, resultaría pertinente incorporar una formación inter-transdisciplinaria.

\section{Palabras clave}

Representaciones sociales - Educación ambiental - Investigación cualitativa - Prácticas pedagógicas.

1- Agradecimientos. La investigación contó con el apoyo del área académica de Diversidad e Interculturalidad de la Universidad Pedagógica Nacional, México.

2- Universidad Pedagógica Nacional, Ciudad de México, México. Contactos: rcalixto@upn.mx; raul_2020@outlook.com. 


\section{Social representations and pedagogical practices in environmental education}

\section{Abstract}

Social representations are a practical knowledge which gives meaning to the environment and sense to different practices. Knowledge of social representations in environmental education is the basis for understanding the practices of environmental educators. The research carried out had the objective of describing the representations of environmental education and the meaning of the pedagogical practices from a sample of environmental educators. It is a descriptive study, of interpretative nature, guided by the process approach to social representations. The results achieved include the observation that environmental educators provide the pedagogical practices in environmental education with a sense towards the conformation of congruent lifestyles with attitudes and values of care and respect to the environment. The research has observed the existence of social representations on environmental education and some of the implications they have in their pedagogical practices. These results confirm why social representations constitute a theory that is increasingly used to understand the common sense found in various educational actors, who can take a stand on pedagogical practice. Likewise, it contributes to interpret the relationships between representations and pedagogical practices in environmental education. Anthropocentric social representations are predominant, so that in the processes of formation and updating of educators, it would be pertinent to incorporate an inter-transdisciplinary training.

\section{Keywords}

Social representations - Environmental education - Qualitative research - Pedagogical practices.

\section{Introducción}

La Tierra está sometida a una creciente presión. En la actualidad se está usando 50\% más de los recursos de los que el planeta puede proveer, a este ritmo, se estima que en el 2030, dos planetas no serían suficientes para satisfacer las necesidades de consumo de alimentos y energía de los seres humanos (WWF, 2012).

La generación Z (posmilénica o centúrica) y siguientes son y serán herederas de los desaciertos de las generaciones anteriores en torno a múltiples aspectos, como el referido a la pérdida de los ecosistemas o el cambio climático antropogénico. En la actualidad el planeta se encuentra en la era del Antropoceno (CRUTZEN; STOERMER, 2000), las actividades ecocidas sobre los bienes naturales están generando cambios irreversibles en los ecosistemas. La educación ambiental constituye una de las alternativas más importantes para abordar, desde el ámbito educativo, los distintos problemas ambientales y 
contribuir a la formación de una conciencia planetaria; es un campo interdisciplinario con implicaciones culturales en las formas de constituirse como una sociedad comprometida con el respeto y valoración del medio ambiente. Dado que la población en general, en la mayoría de los países, manifiesta un desconocimiento de las consecuencias hacia el medio ambiente de las actividades extractivas, productivas y de circulación de los bienes naturales en el mercado con base a la energía proveniente de combustibles fósiles; el papel de los educadores ambientales debe ser más fuerte que nunca al "[...] mantener una actitud abierta y flexible a la integración de diversas metodologías y estrategias de intervención para mejorar la relación de las personas con su entorno" (CASTR0, 2000, p. 375).

Un educador ambiental, en su quehacer cotidiano, desarrolla un conjunto de prácticas orientadas a diversos propósitos como son el comunicar, informar, sensibilizar, así como de generar cambios de comportamiento, de actitudes o de valores. En este sentido, Meira argumenta que la materia prima de un educador ambiental en el último término es “[...] la forma en que nos representamos dentro del mundo -y no sólo el mundo como entorno o medio externo-, y la forma en que nos relacionamos" (2005, p. 272).

La tarea de un educador ambiental contribuye a que los seres humanos representen el medio ambiente de tal forma que logren observarse como parte de éste, como actores conscientes y activos, conocedores de sus derechos y responsabilidades ambientales y capaces de actuar en consecuencia. Al respecto, Palmer (1992) refiere la importancia de realizar investigaciones en torno a la figura del educador ambiental, con lo cual se pueda revisar la influencia de las experiencias del educador en el desarrollo de personas bien informadas y ambientalmente activas; por otra parte, Paige, Lloyd y Chartres (2008) señalan la importancia de conocer la influencia de los entornos educativos en la formación de los educadores ambientales.

Al igual que en otros países, en México existe un creciente interés por incidir en la construcción de una ciudadanía ambiental, interesada en atender los diversos problemas ambientales de nuestro tiempo. Existen varios programas formativos, abocados a la formación de educadores ambientales (GONZÁLEZ-GAUDIANO; ARIAS, 2015), sin embargo, no todos los educadores ambientales de México son egresados de alguna institución superior, ni todos los egresados de un programa en educación ambiental se asumen como tales.

En este marco, surge el interés de realizar una investigación que tiene como objetivo describir las representaciones de educación ambiental y del sentido de las prácticas pedagógicas de una muestra de educadores ambientales.

\section{Representaciones sociales y prácticas pedagógicas en educación ambiental}

Moscovici (1979) dio a conocer la teoría de las representaciones sociales, en la que se plantea un sistema de interpretación integrado por significaciones individuales y sociales que da forma a la estructura coherente de un objeto. Las representaciones sociales pueden entenderse como un conjunto de principios construidos interactivamente y compartidos por un grupo con el cual le dan sentido y significado a la realidad, respecto al medio 
ambiente pueden encontrase representaciones sociales antropocéntricas, globalizantes y naturalistas (REIGOTA, 1995). En las representaciones sociales antropocéntricas se centra la atención en los beneficios al ser humano; en las globalizantes se priorizan las relaciones sociedad naturaleza y en las naturalistas sólo se toma en cuenta a los componentes, procesos y fenómenos naturales.

En los últimos años, en México ha ocurrido un aumento en el número de investigaciones sobre las representaciones sociales en diversos tópicos de la educación, como la formación docente, la práctica educativa, excelencia académica, educación ambiental, entre otros. En la actualidad resulta difícil precisar el número y diversidad de temas que son investigados por medio de las representaciones sociales. Al respecto, Cuevas y Mireles (2016) hacen una revisión de investigaciones que utilizan las representaciones sociales, encontrando 141 trabajos que abordan diversos tópicos: práctica docente (16\%); política educativa (13\%); educación ambiental (11\%); instituciones escolares (8\%); formación e identidad profesional (7\%); sujetos (profesores, orientador, autoridades universitarias, niños) (6\%); formación docente (6\%); prácticas escolares de los estudiantes (6\%); trayectoria escolar (6\%); enseñanza de las matemáticas (6\%); el currículo (5\%); evaluación educativa en diferentes modalidades: institucional, docente y del aprendizaje (4\%); construcción social de la ciudadanía y los valores (4\%); y otros (8\%) en que se abordan temas emergentes como género, inclusión educativa y tecnologías de la información y la comunicación. Uno de los supuestos derivados de los estudios realizados vincula la posibilidad de una mejor comprensión de prácticas en los diversos actores educativos a partir del reconocimiento de las representaciones sociales; con lo que emerge la posibilidad de realizar diferentes adecuaciones a las planeaciones de acuerdo con las representaciones sociales de los actores educativos a partir de las singularidades de los conocimientos de sentido común. Además, se develan las relaciones entre las representaciones y las prácticas, en un espacio institucional, social o cultural. El 11\% de investigaciones referidas a las representaciones sociales en educación ambiental, identificadas por Cuevas y Mireles (2016), tiene como sujetos de estudio a estudiantes de educación superior, estudiantes de educación normal y profesores de educación primaria, pero no reportan algún estudio realizado con educadores ambientales.

Para Morera y otros autores (2015), las representaciones sociales corresponden a una red de conceptos e imágenes interactivas, porque condensan significados y constituyen sistemas de referencia que permiten interpretar y clasificar una realidad, dotando de un sentido a las acciones cotidianas. Los significados de las representaciones sociales se comparten a través de las prácticas sociales, las cuales son consideradas modos de acción, que pueden ser formas espontáneas u organizadas, individuales o grupales; formales e informales.

Las representaciones y las prácticas sociales son un producto de las relaciones humanas, que se manifiestan a través de las interacciones cotidianas que ocurre en el grupo. Las prácticas construyen representaciones en un proceso histórico, pero las representaciones determinan ciertas prácticas a través de un movimiento dialógico entre ambos procesos.

No hay una relación determinista entre las representaciones y las prácticas sociales. Así como un conjunto de prácticas puede asociarse a una representación, un conjunto de representaciones puede asociarse a una práctica. 
Antes de las prácticas, las representaciones desempeñan un papel esencial, pero también intervienen después de la práctica, para explicar y justificar los comportamientos. Una de las funciones que se les atribuye a las representaciones sociales es el de orientación de la práctica, ya que pueden constituir una guía de comportamientos y prácticas, es decir una guía para las prácticas, entre otras para las prácticas pedagógicas en educación ambiental.

De acuerdo con Abric (2001), la representación interviene directamente en la definición de la finalidad de la situación; también produce igualmente un sistema de anticipaciones y expectativa, con lo cual se seleccionan y filtran las informaciones e interpretaciones con objeto de volver esa realidad conforme a la representación. Las representaciones sociales pueden ser prescriptivas de comportamientos o prácticas obligadas.

Las representaciones sociales son diferentes en cada grupo, dependen de múltiples factores, como los papeles que se desempeñan, las experiencias de vida, el nivel de formación escolar; sin embargo, diferentes grupos, de acuerdo con la información que reciben por distintos medios y las comunicaciones que se produzcan, van adquiriendo algunos elementos en común.

Por lo anterior, los conocimientos de las representaciones sociales de la educación ambiental de los educadores ambientales constituyen la base para la comprensión de su práctica pedagógica, en un momento y espacio determinado. La representación social les permite a los educadores ambientales ubicarse en el mundo social, ordenar las ideas y guiar sus tareas profesionales. La práctica pedagógica se configura como una actividad esencialmente ética, cuyo sentido y significación es construido histórica y socialmente (GIMENO; PÉREZ, 1992; FREIRE, 1998).

En la trayectoria de la educación ambiental se han desarrollado diversos enfoques, orientados por la educación popular (REYES, 1984), la ecopedagogía (GADOTTI, 2003), la economía verde (O'DONOGHUE; RUSSO, 2004), los principios de la naturaleza (LOUV 2011), la socioecología (KYBURZ-GRABER, 2013), el consumo verde (BARENDREGT; RIVKE, 2014), las cosmovisiones de los grupos originarios (TUCK; MCKENZIE; MCCOY, 2014), el desarrollo sostenible (SCOTT, 2015), la educación para un futuro sostenible (PAVLOVA, 2015), la ecociudadanía (SAUVÉ, 2017), la integración de la cultura con la naturaleza (SANDERS; RYKEN; STEWART, 2018), la pedagogía crítica (PIASENTIN; ROBERTS, 2018), el cambio climático (GONZÁLEZ- GAUDIANO; MEIRA, 2020), entre otros. Algunos de estos enfoques han sido impulsados por organismos internacionales como la UNESCO, y otros por los propios educadores.

En cada uno de los enfoques se encuentran presentes las representaciones sociales de la educación ambiental, delineando diversas prácticas pedagógicas. En las prácticas se encuentran implícitas las finalidades de la educación ambiental que se pretende desarrollar, trazan el camino o caminos por seguir y llevan a cabo las estrategias y acciones para lograr o fomentar los conocimientos, los comportamientos, las actitudes y/o valores deseados.

Las prácticas pedagógicas se aprenden con los otros: en un grupo, en la comunidad a la que el educador desea adherirse y desea aprender su conocimiento sistemático y sus patrones de conocimiento en la acción (SCHÖN, 1998), se establecen interacciones en esta comunidad, en donde se comunican, entre otros aspectos, las representaciones sociales de la educación ambiental. Las prácticas pedagógicas en educación ambiental 
se manifiestan de múltiples formas: en habilidades, en el lenguaje, métodos, materiales, valores, competencias, entre otros. Requieren, de acuerdo con Sauvé (2013), del desarrollo de tres campos de competencia: competencia crítica, competencia ética y competencia política. Competencias que se manifiestan según varios factores, como las condiciones del contexto, los ámbitos de actuación, los sujetos participantes, las modalidades de atención y los problemas ambientales emergentes entre otros.

Por otra parte, aunque existen paralelismos, en cuanto a los fines de las prácticas pedagógicas de los educadores ambientales y de los ecologistas o defensores ambientales en los movimientos ambientales, los alcances son diferentes, aún falta mucho por conocer en cuanto a las relaciones entre sus prácticas (ALVES et al., 2017).

\section{Metodología}

La investigación tiene una orientación cualitativa, recupera las propias palabras de las personas generadas de forma oral y escrita. Para Hart (2007) esta orientación recupera las narraciones de los entrevistados para considerar las experiencias de los educadores. La investigación realizada se dirige a la interpretación de las representaciones de educación ambiental y del sentido de las prácticas de un grupo de personas (educadores ambientales), por lo cual se delimita como un estudio descriptivo, de carácter interpretativo, orientado por el enfoque procesual de las representaciones sociales. Es un estudio descriptivo porque muestra los componentes de las representaciones sociales que posee un grupo de educadores ambientales; es un estudio interpretativo porque interesa lo particular y lo contextual de las representaciones, se comprende la realidad como dinámica y diversa.

El proceder metodológico tiene una vía inductiva-deductiva dando la posibilidad de favorecer la valoración y apreciación de las personas involucradas en los procesos educativos. Se propone reconocer la valoración de las experiencias de los educadores ambientales, cuyo propósito es no solo incidir en las presencias y ausencias que se han producido alrededor de la cotidianeidad de la práctica. Es decir, en las representaciones y en las prácticas se identifican las características, con ausencias, problemas, retos, avances y logros.

Moscovivi (1979), al igual que otros autores como Jodelet (1986), propone una perspectiva multi-metodológica para el estudio de las representaciones sociales. En esta investigación se emplean la encuesta y la entrevista cualitativa, cada una permite identificar los diversos componentes de las representaciones de la educación ambiental y las prácticas pedagógicas. La encuesta tuvo el objetivo de identificar la existencia de representaciones sociales de la educación ambiental. Se elaboró un cuestionario con preguntas abiertas (CUEVAS, 2016) con aspectos de las tres dimensiones de las representaciones sociales (información, campo de representación y actitudes) y se complementó con dos cartas de asociaciones libres (ABRIC, 2001), en una de éstas se propuso como término inductor educación ambiental y en la otra el término de prácticas pedagógicas. Con el cuestionario se produce un discurso susceptible de análisis; en las respuestas de las preguntas abiertas se encuentran varios elementos en los que los educadores expresan lo que saben, lo que observan y lo que sienten en torno a la educación ambiental. Y en la asociación libre, los educadores ambientales generan las palabras que a su juicio tienen relación con 
los términos inductores, con lo cual es posible obtener los elementos constitutivos del contenido de la representación.

Las categorías que emergieron del análisis de las respuestas de las encuestas son: el significado de la educación ambiental, el sentido de las prácticas pedagógicas y las representaciones como generadoras de prácticas pedagógicas. Con base en estas tres categorías se construyó el guión de entrevista semiestructurado, y se registran y transcriben los relatos de los educadores a fin de reconstruir sus experiencias en torno a la educación ambiental y a las prácticas pedagógicas. El análisis de la información de la encuesta y la entrevista implicó varios procesos, organización, codificación, agrupación por temas emergentes, al encontrar paralelismos se definieron las categorías (CRESWELL, 2013). El análisis de las entrevistas inició con la identificación de los términos recurrentes, después se agruparon de acuerdo con su grado de similitud, se formaron grupos y se les asignó un nombre a las categorías.

La muestra fue selectiva, no probabilística, con base en dos criterios de inclusión de los participantes del estudio: experiencia en el campo de la educación ambiental y con una o más publicaciones referidas al campo en los últimos tres años.

En los primeros meses del año 2018 se invitó a 20 educadores a participar en la investigación; educadores con quienes se había interactuado en el año de 2017, al coordinar dos libros Educación ambiental en las instituciones de educación superior (CALIXTOFLORES; MORENO, 2017) e Investigaciones y prácticas pedagógicas en educación ambiental (CALIXTO-FLORES, 2018). A los educadores se les explicó los objetivos y el proceso de desarrollo del estudio, y trece aceptaron participar. Los cuestionarios de la encuesta fueron enviados a sus correos electrónicos, y de forma posterior se realizaron las entrevistas vía Skype, tomando el común acuerdo de grabar las conversaciones.

Los participantes en el estudio radican en distintos estados de la república mexicana: Michoacán, San Luis Potosí, Chihuahua, Coahuila, Estado de México, Oaxaca, Baja California Norte, Veracruz, Ciudad de México, Nuevo León y Yucatán.

En la presentación de las características de la población de estudio se anota entre paréntesis la frecuencia.

- La población femenina (10) es la predominante.

- El promedio de edad es de 43 años.

- La mayoría (11) tiene estudios de doctorado: Educación Ambiental (1), Ciencias de la Educación (2), Ciencias Ambientales (1), Química (1), Administración y Educación (1), Investigación Educativa (3), Educación (2).

- Una educadora ambiental obtuvo el grado de maestra en Desarrollo rural, con especialidad en Educación.

- Un educador ambiental tiene la formación como ingeniero en Horticultura.

- El promedio de horas que dedican a la semana a la educación ambiental es de 28 horas y 12 horas a las actividades relacionadas con la investigación.

- Un poco menos del tercio de participantes (5) pertenecen a una red o asociación relacionada con la educación ambiental (Red de Educadores Ambientales de Coahuila; Red de Investigadores de Educación para el Desarrollo Sustentable; Joint Task Force on 
Forest; Red de Patrimonio Biocultural y CoLaboratorio de Oceanografía Social; Academia Nacional de Educación Ambiental).

- El mayor número de educadores ambientales (11) se encuentra laborando para una institución de educación superior. Un educador además de impartir docencia en diversos centros de educación ambiental participa activamente en un movimiento ambientalista. Y una educadora que se ha desempeñado como docente en distintos niveles educativos y en la actualidad concentra sus actividades como directora de un instituto de investigación educativa.

- El promedio de años que se han dedicado a la educación ambiental es de 9.5 años.

- El lugar de residencia y laboral, corresponde a distintos Estados de la república mexicana: Baja California Norte (1), Chihuahua (2), Coahuila (1), Nuevo León (1), San Luis Potosí (1), Oaxaca (1), Ciudad de México (1), Estado de México (1), Michoacán (1), Veracruz (1) y Yucatán (2).

Los resultados de la entrevista comprenden respuestas textuales, para identificar al educador ambiental, se anota entre paréntesis las iniciales que corresponden al Estado de la república mexicana donde radican, en los casos de Chihuahua y Yucatán acompañado de un número: Michoacán, Mn; San Luis Potosí, SLP; Chihuahua, Ch1 y Ch2; Coahuila, Ca; Estado de México, EM; Oaxaca, Ox; Baja California Sur, BCS; Veracruz, Vz; Ciudad de México, CdM; Nuevo León, NL; Yucatán, Yn1 y Y2.

\section{Resultados}

En la presente investigación, como resultado de la encuesta fue posible identificar los principales componentes de las representaciones y organizarlos en tres niveles, de acuerdo con la frecuencia en los que son expuestos. El primer nivel corresponde al núcleo figurativo de la representación, son los elementos que son mencionados recurrentemente en el discurso; en el segund nivel, se ubican los elementos que son mencionados en menor frecuencia; y en el tercen nivel, figuran los elementos que son mencionados circunstancialmente y que aparecen asociados a algunos de los elementos que le anteceden.

Primer nivel: interdisciplinario, responsabilidad, respeto, integradora, complejidad, de campo, hábitat, transdisciplinariedad, problemas ambientales, medio ambiente, comprensión, propositiva, transcampo, generación de conocimiento, búsqueda de soluciones y mejora de las prácticas pedagógicas.

Segundo nivel: trinomio sociedad-ambiente-economía, compromiso, enriquecedora, cualitativa, uso irracional, naturaleza, equilibrio realidad social, multidisciplinar, formal y no formal, oportunidades, holística, crítica / transformadora, problematización, interdependencia, contexto, conciencia, ética social, prácticas, comunidades, múltiples métodos.

Tercer nivel: prospectivo, resultados, flexible, procesual/relacional, efectos, ecología, sustento teórico educativo, filosofía de vida, experiencia, incertidumbre, cosmovisión, preventiva, holístico, apoyo, aplicable, incluyente, estado del arte, cuantitativa, sustentabilidad, evaluación, convivencia, independiente de la escuela, estratégico, estímulos, visión de futuro, propuesta de mejora, sensibilidad, incertidumbre, coherencia interna, práctica, método, soluciones, injusticias, delimitación, cambio, tranformación, comunidades sociales. 
Los elementos organizados en los tres niveles dan cuenta de la existencia de las representaciones sociales de la educación ambiental, en las que es posible reconocer los tres tipos de representaciones propuestas por Reigota (1995): antropocéntricas, globalizantes y naturalistas. Así, por ejemplo, los participantes en el estudio expresan que la educación ambiental tiene una estrecha relación con los problemas ambientales originados por acciones irracionales; en este marco la educación ambiental tiene como finalidades la generación de conocimiento, la búsqueda de soluciones a los problemas ambientales y mejora de las propias prácticas. En las representaciones identificadas en los educadores predominan las representaciones sociales antropocéntricas del medio ambiente; por lo que, como lo refiere Jacobi (2005), los educadores deben ser cada vez más mejor preparados para poder identificar las múltiples determinaciones e interacciones de las dimensiones del medio ambiente.

La educación ambiental como campo interdisciplinario se encuentra estrechamente vinculada con otros campos, tiene interconexiones con disciplinas de las Ciencias Sociales, Humanas, Naturales y Exactas. Sin embargo, sus contornos abarcan aspectos ambientales y educativos, acordes a su estructuración interna. Las representaciones de la educación ambiental permite identificar el sentido de las prácticas pedagógicas, las cuales poseen elementos en común.

Como resultado del análisis de la encuesta, emergen las categorías con las que se construyeron las entrevistas: significado de la educación ambiental, sentido de las prácticas pedagógicas y representaciones como generadoras de prácticas pedagógicas.

\section{Significado de la educación ambiental}

Moscovici (1979) delimita una de las principales funciones de las representaciones sociales. En su capacidad para crear información, ayuda a la familiarización con lo extraño a partir de las categorías presentes en el conocimiento de sentido común. Esta función denota la importancia de esta teoría para la investigación educativa, ya que las representaciones sociales presentes en el mundo cotidiano influyen en la conformación de las prácticas pedagógicas.

En el discurso de los educadores ambientales es posible identificar los contenidos anclados en el pensamiento social de los educadores, resultado de tradiciones y prácticas desarrolladas en su formación académica y desarrollo profesional, de los conocimientos de sentido común que se comunican cotidiamente entre sus colegas; “[...] hacemos de la ciencia un bien comestible como cualquier otro (MOSCOVICI; HEWSTONE, 1986, p. 680). Es decir, las representaciones se incorporan un esquema de pensamiento preexistente. Para lograrlo se da simultáneamente un proceso de objetivación, en el cual lo abstracto se torna concreto a través de imágenes (selección y descontextualización, formación de un núcleo figurativo y naturalización). Los resultados de esta categoría se organizan en dos grupos: la educación ambiental en la construcción del conocimiento y la educación ambiental en la búsqueda de soluciones a los problemas ambientales.

- La educación ambiental en la construcción de conocimiento.

Aprehensión de conocimiento aplicable a la comprensión del medio ambiente. (0x). 
Generación de conocimiento que permita comprender y tomar decisiones sobre los diversos procesos y momentos de la educación ambiental. (Y1).

Construyen nuevas formas de organizar el conocimiento. (Cd.M).

Conocimientos para las comunidades, enfrentar los conflictos y defender sus territorios. (BCS)

Busca la rearticulación de los conocimientos ambientales. (Ca).

- La educación ambiental en la búsqueda de soluciones a los problemas ambientales.

Es un proceso de indagación acerca de la búsqueda de soluciones a los problemas ambientales. (Mn). La búsqueda, la indagación de soluciones a problemas existentes, pero también vinculados a la historia a lo que hemos hecho y sus consecuencias. (SLP).

Proceso por el cuál se identifican y plantean soluciones a los diversos problemas relacionados con la educación ambiental, y lo que ella implica. (Ca).

Es un proceso complejo, conciente y sistemático donde el educador tiene por objeto de estudio el análisis del medio ambiente: su utilización, abuso desmedido, situación actual, efectos en la salud humana y sobre todo la reflexión de propuestas para su mejora del cuidado y preservación. (EM). Proporciona las bases que contribuyen a identificar las necesidades de la población en materia de educación ambiental con la finalidad de colaborar con soluciones a los diferentes problemas ambientales del entorno. (Vz).

Payne (2020) plantea una crítica a la construcción del conocimiento ambiental y a la práctica pedagógica, ya que no se ha dado solución a sus fundamentos axiológicos, epistemológicos y metodológicos, porque se ha privilegiado el discurso de la educación ambiental proveniente de los países del hemisferio del norte.

Los educadores ambientales, participantes en el estudio, no significan a la educación ambiental en torno al discurso dominante de la sustentabilidad, sino a partir de los problemas ambientales. Los educadores ambientales expresan sus preocupaciones por los problemas ambientales de su localidad (tala de árboles, desertificación, contaminación, ente otros). En este sentido, Meira (2013) señala que los educadores ambientales cada vez más se encuentran implicados en la tarea de desactivación simbólica de estos problemas. Aunque pocos educadores ambientales participantes en el estudio se perciben implicados en los problemas ambientales globales, como degradación de la capa de ozono y el cambio climático.

En esta categoría es posible identificar objetivos comúnes en las prácticas de los educadores ambientales, entre estos objetivos se encuentran la construcción de conocimientos y la búsqueda de soluciones para los problemas ambientales, objetivos que son reconocidos tambén por Kyburg-Graber (2013) en el enfoque socioecológico, ya que considera que los sujetos pueden generar conocimiento sobre problemas ambientales complejos si tienen la oportunidad, con los cuales pueden desarrollar acciones para incidir en el cambio de situaciones presentes en su vida cotidiana. Por otra parte, Allen y Crowley (2017) coinciden con este planteamiento y proponen prácticas que tomen en cuenta la participación activa, la pertinencia y la interconexión de experiencias. Se reconoce la importancia de la construcción del conocimiento, de las experiencias y de los conocimientos previos para lograr incidir en la realidad socioambiental. Aunque existen factores que no han sido tomados en cuenta cuando la educación ambiental se orienta en la búsqueda de soluciones a los problemas 
ambientales, Stevenson (2007) refiere que las prácticas pedagógicas en educación ambiental llegan a confrontarse con las prácticas dominantes en las escuelas, que generalmente no implican un cuestionamiento de los problemas ambientales, al limitarse al aprendizaje pasivo. Por ello, en las instituciones educativas permea el significado de educación ambiental como sinónimo de ecología, un significado parcial, al no tomar en cuenta las otras disciplinas que estudian al medio ambiente.

\section{El sentido de las prácticas pedagógicas}

Los educadores son actores sociales con preferencias particulares, gustos, anhelos, temores, simpatías, sentimientos, repulsiones, frustraciones, etc. que imprimen un sentido a las prácticas que realizan, las temáticas que trabajan y perspectivas que utilizan, permitiéndonos prever la postura que asumen ante la investigación en educación ambiental. Al respecto, Abric (2001) señala que las representaciones sociales constituyen una guía para la acción debido a que intervienen directamente en la definición de la finalidad de la situación, produce un sistema de anticipaciones y expectativas, así como es prescriptiva de comportamientos. Estos factores referidos por Abric (1987) permiten comprender las relaciones entre las representaciones y prácticas de la educación ambiental. Las prácticas pedagógicas se reflejan en una serie de acciones, actitudes y disposiciones en las que se articulan historia y memoria colectiva con procesos que se dan en el tiempo histórico actual (NIEVES, 2014). En esta categoría se forman dos grupos: la transformación de las relaciones con el medio ambiente y obstáculos a las prácticas pedagógicas.

- La transformación de las relaciones con el medio ambiente.

Contribuye a que las personas conozcan las interrelaciones con su entorno. (Ch1).

Se adquieren herramientas destinadas a generar cambios en sus forma de vida que permitan la posibilidad de vivir en ambientes sustentables. (NL).

Propicia el cambio favorable de prácticas ambientales. (0x).

Persigue la comprensión de las acciones de los agentes del proceso educativo a favor del ambiente. (Ch1).

Es aquella que pretende favorecer un cambio en las relaciones de las personas con el medio por medio de la identificación de los factores que pueden frenar los problemas que afectan al ambiente, a la humanidad y desarrollen políticas a favor del mismo. (Y2).

- Obstáculos a las prácticas pedagógicas.

Aún prevalece poca reflexión y poco espacio para que la comunidad se involucre en el desarrollo de estas prácticas, sigue siendo muy existencialista y muy patriarcal y eso lo hace que sea poco crítica, poco participativa. (BCS).

Desconocimiento en la comunidada académica del "status" científico de las ciencias sociales y humanas. (SLP). 
Prevalencia de una perspectiva conservacionista en la educación ambiental que se refleja en el predominio de temas referidos al cuidado del medio ambiente. (Mn).

Confusiones entre los aspectos que comprenden la ecología con los de la educación abiental. (Vz). Pocas posibilidades de diálogo con otros colegas respecto a temas y/o problemas de la educación ambiental. (Y2).

En esta categoría se observa que el sentido que se les confiere a las prácticas pedagógicas ocurre por los alcances y limitaciones que los educadores ambientales reconocen en la educación ambiental. En los educadores ambientales predominan las representaciones sociales antropocéntricas, ya que dirigen las prácticas hacia el estudio de los impactos del medio ambiente en relación a la supervivencia del ser humano, se observa una tendencia hacia el enfoque crítico. Este enfoque, de acuerdo con Piasentin y Roberts (2018), se orienta hacia el cuestionamiento de la realidad ambiental, el análisis y construcción de alternativas de acción.

Los educadores ambientales propician el aprendizaje experiencial, la reflexión sobre los problemas desde distintas perspectivas con una visión crítica y transformadora. El sentido de las prácticas pedagógicas en educación ambiental orientadas por el enfoque crítico da la posibilidad de reconocer las implicaciones de su práctica en la conformación de aprendizajes congruentes con actitudes y valores de cuidado y respeto al medio ambiente. Aunque en la muestra pocos educadores ambientales toman en cuenta las desigualdades sociales del contexto donde desarrollan su práctica, la mayoría centra su atención en los efectos negativos a la salud humana, perspectiva congruente con el predominio de las representaciones sociales antropocéntricas. Esta situación, según Hursha, Henderson y Greenwood (2015) se presenta porque los propios educadores están implicados en cuestiones de economía, por lo que no se alcanza a visualizar la legimitación de las diferencias del consumo pesonal. Ya que como lo mencionan 0’Donoghue y Russo (2004), la educación ambiental se encuentra implicada en la producción y legitimación del conocimiento ambiental, impregnando en la práctica las formas de pensar (representaciones sociales) de los educadores al implementar y desarrollar la educación ambiental.

La mayoría de los educadores coincide en señalar la importancia de la educación ambiental, pero encuentran, como uno de sus principales obstáculos, el escaso interés institucional por apoyar sus proyectos y la persistencia de ecologizar los programas educativos, es decir, en los programas institucionales no se toma en cuenta la dimensión social del medio ambiente.

\section{Las representaciones como generadoras de las prácticas pedagógicas}

Las representaciones sociales sobre la educación ambiental proporcionan los elementos para identificar las prácticas pedagógicas. Una práctica comprende “[...] la concretización a la simple intención, el acto al pensamiento y, de otro lado, el hábito a lo esporádico o lo novedoso" (ROUQUETTE, 2000, p. 138).

Las prácticas se concretan en una o en una serie de acciones, es decir, la representación que se tiene en la mente de la práctica pedagógica en educación ambiental se manifiesta 
en el acto, en el hacer. De acuerdo con Castro (2000), estas prácticas pueden clasificarse según el método: directo e indirecto. Los métodos directos, ponen en contacto directo a las personas con las situaciones reales del medio ambiente; en tanto que en los indirectos se llevan a cabo acciones que acercan a las personas a las situaciones del medio ambiente con información y argumentos fundamentados para el cambio de comportamientos, actitudes y valores. Las acciones de estos métodos se pueden combinar y desarrollarse en la formación y capacitación, en la información y comunicación y en la participación ambiental. Para Jacobi (2005) las prácticas educativas en educación ambiental se han de orientar al cambio de hábitos, actitudes y prácticas sociales, así como al desarrollo de habilidades, evaluación y capacidad de participación de estudiantes.

En este marco emerge la importancia de identificar las relaciones entre las representaciones y las prácticas en educación ambiental; cada educador otorga determinado sentido a las prácticas pedagógicas de acuerdo con las representaciones sociales que posee sobre la educación ambiental. En esta categoría, la información se organiza según tres grupos: procesos de concientización, prácticas ante los problemas ambientales y acciones y formas de actuación.

\section{- Procesos de concientización.}

La educación ambiental es un proceso que permite la toma de conciencia sobre el valor del medio ambiente. (SLP).

Es un proceso que propicia la construcción de conocimiento de las relaciones socioambientales considerando el espacio- tiempo sociohistórico donde se dan dichas relaciones; su finalidad es concientizar sobre la problemática ambiental y encontrar soluciones sustentables a dicha problemática. (Ca).

Se desarrollan saberes y conocimientos referentes al cuidado y preservación del medio ambiente, de ahí que pretende crear conciencia a las nuevas generaciones sobre su importancia y trascendencia a fin de que integren en su actuar cotidiano cierta reflexión para la utilización y consumo. (EM).

Desarrollar una conciencia ambiental no nada más en los jóvenes, en los profesionistas, en todas personas y que no nada más se quede en una conciencia ambiental, sino que se refleje en acciones urgentes. (Ch1).

Propicia la conciencia a la comunidad educativa de los problemas ambientales y de sus impactos a la humanidad. (Mn).

- Prácticas para el conocimiento del medio ambiente.

Desarrollo y ejecución de proyectos educativos relacionados con el medio ambiente-contaminación y actitudes ambientales. (Ch1).

Se necesita gente que entusiasme a los demás, los motive, sobre todo a los muchachos, que los motive y pues ellos están dispuestos a participar, entonces creo que lo que falta es eso, difundir el trabajo que se hagan más actividades significativas. (SLP). 
Un proceso de enseñanza-aprendizaje de conocimientos y actitudes orientados a mostrar el funcionamiento de los ambientes naturales y cómo los humanos podemos respetar y coexistir de un modo sustentable. (Mn).

Se generan nuevas actitudes y valores que los lleve a ejecutar acciones de cuidado por y respeto por la naturaleza y el medio. (Ch2).

Diseñar estrategias sobre el proceso de aprendizaje en temas medioambientales. (CdM).

- Acciones y formas de actuación ante los problemas ambienales.

Mi práctica tiene que ver con identidad y territorio, y sobre todo con identidad y defensa del territorio, porque yo veo como un reto súper fuerte a todas las prácticas sobre todo de educación ambiental con los temas de resistencia con los mega proyectos, como agricultura monointensiva, minerías, y aquí en Baja California con el desarrollo turístico hotelero. (BCS).

Las acciones se dirigen a la identificación y resolución de situaciones que afectan el ambiente desde una perspectiva global. (NL).

Acompañar a los estudiantes en el proceso educativo que les permita descubrir diferentes problemas medioambientales y sus causas y consecuencias. (BCS).

Propicia la construcción conjunta de soluciones a los problemas ambientales detectados. (Y1).

Trabajando hacia la concientización en el manejo de residuos sólidos y en el manejo del cuidado del agua, podríamos trabajar cuestiones de formación en cuanto a programas o proyectos que tuvieran que ver con el cuidado de la fauna, la flora. (Y2).

Entre las prácticas pedagógicas desarrolladas por los educadores ambientales se encuentran los proyectos escolares o comunitarios, con acompañamiento del educador. Prácticas como las que refieren Monroe y otros autores (2017), orientadas a la concientización. Con estas prácticas se favorece la obtención de conocimientos, pero también comprensión de los orígenes de los problemas ambientales, y se promueve la participación activa.

La mirada de los educadores ambientales es amplia, al significar las interrelaciones de las acciones locales, regionales y globales para incidir en un cambio de mentalidad en torno a las relaciones del ser humano con el medio ambiente, aunque son escasas las referencias sobre el cambio climático. Según Krasny y Dillon (2012), se requiere abordar en la práctica las interconexiones entre distintas disciplinas, con un enfoque socioecológico. Los educadores ambientales se inscriben en este enfoque cuando buscan la interdisciplinaridad al retomar en su práctica aspectos de las Ciencias Naturales, Ciencias Sociales y Ciencias Humanas, ninguno de los educadores de la muestra confunde a la educación ambiental con la ecología.

Las prácticas pedagógicas parten del reconocimiento de una necesidad de atender desde una mirada educativa los problemas ambientales, por lo que los educadores vinculan sus prácticas con los problemas ambientales. Al respecto, Stevenson (2007) destaca la importancia de esta vinculación, abordando problemas específicos acordes al desarrollo cognitivo y experiencial de los estudiantes, se retoman problemas locales, sin perder de vista los porblemas globales. Las representaciones como generadoras de las 
prácticas pedagógicas orientas a la concientización, solución a los problemas ambientales y acciones y formas de actuación.

Se deben de evitar suposiciones unilaterales de las prácticas pedagógicas de educación ambiental. Existe una limitación en los alcances, el comportamiento y la motivación humana son enormemente complejos como lo refieren Heimlich y Ardoin (2008), por lo que las acciones y formas de actuación de los estudiantes no son siempre las esperadas. Según los autores, se hace necesario que los educadores ambientales comprendan que las personas no se encuentran motivadas por las mismas cosas, ni son igualmente capaces de cambiar sus hábitos y compartamientos, y aún es más complicado en incidir en la transformación de sus estilos de vida. Lo cual implica un reto para los educadores ambientales: el de replantearse constantemente su práctica pedagógica de acuerdo a las necesidades, problemas y expectativas de sus estudiantes.

\section{Conclusiones}

Estos resultados confirman porque las representaciones sociales constituyen una teoría que cada vez más se utiliza para comprender los conocimientos de sentido común que poseen los diversos actores educativos, los cuales pueden tomar una postura en torno a las practicas pedagógicas. De igual forma, contribuyen a interpretar las relaciones entre las representaciones y prácticas pedagógicas en educación ambiental. A lo largo de la investigación realizada, los educadores en diferentes momentos confieren el sentido de las prácticas pedagógicas a la conformación de una conciencia ambiental en las personas, a través del desarrollo de diversas estrategias y acciones: llevan a cabo prácticas que confrontan a las prácticas dominantes impulsadas por los organismos internacionales.

Todos los educadores ambientales consideran a la concientización como una de las principales finalidades de las prácticas pedagógicas en educación ambiental; para ellos las prácticas se delimitan en un actuar reflexivo y consciente ante los diversos problemas del medio ambiente. Las acciones de los educadores ambientales no solo se dirigen a los componentes cognitivos, son de igual importancia los componentes valoral y actitudinal.

En las representaciones sociales ocurren simultáneamente procesos interrelacionados de percepción, categorización y significación. La percepción determinada por la difusión y divulgación de los conocimientos científicos; la categorización de los distintos componentes que la constituyen; y la significación otorga el sentido a participar o compartir la representación. Las representaciones sociales de los educadores ambientales tienen su origen en diversas experiencias de vida. Las representaciones y prácticas se retroalimentan de una forma constante, son generadas en las interacciones en la vida cotidiana, surgen de ella como la manifestación del interior de los sujetos. A través de la comprensión de las prácticas, se puede entender cómo se conforman las relaciones entre los integrantes de diversos grupos.

En este sentido, las representaciones sociales de las prácticas pedagógicas desarrolladas por los educadores ambientales son el resultado de la intersección de lo individual y lo social. Las representaciones sociales de los educadores ambientales dependen de múltiples factores, como las actividades remuneradas que se desempeñan, las 
experiencias de vida, el nivel de formación escolar. Sin embargo, las primeras experiencias de vida son fundamentales en la toma de decisiones de los educadores ambientales. Las personas, en una sociedad tan compleja como la actual, toman la decisión de involucrarse en determinadas actividades por múltiples factores, entre los que se encuentran la conformación de las representaciones sociales sobre diversos objetos. En el caso de los educadores ambientales, estas representaciones son sobre las relaciones de los seres humanos con el medio ambiente, y del papel de la educación ambiental para transformar estas relaciones. De cierta forma, se ha observado en los educadores ambientales, la representación social de que a través de la educación ambiental se puede sembrar la semilla del cambio, con la conformación de una conciencia ambiental.

Los educadores ambientales se inscriben en el campo de la educación ambiental porque se identifican con las finalidades y acciones de esta educación, y construyen prácticas pedagógicas, desarrollando estrategias y acciones fundamentadas en el estudio de las relaciones entre la educación y el medio ambiente. Sin embargo, hay que tomar en cuenta que las representaciones se despliegan en las prácticas, donde se puede observar el significado y sentido que imprimen a las relaciones de la sociedad con el medio ambiente.

En la gran diversidad de enfoques existentes en la educación ambiental, los educadores ambientales participantes en la investigación les confieren un sentido similar a las prácticas pedagógicas en educación ambiental, predominan las representaciones sociales antropocéntricas, comparten características similares en torno a la importancia de los procesos para la conformación de conocimientos, actitudes y valores. La era del Antropoceno no solo implica aspectos geológicos o ambientales, también comprende un plano ideológico, en el cual los educadores ambientales no logran identificar con claridad sus repercusiones globales. Es por ello que la mayoría de los educadores centran su atención en los problemas ambientales locales, y no en los globales y en los efectos negativos de estos problemas en la salud humana. Las prácticas pedagógicas son diversas, corresponden a los enfoques que le imprimen a la educación ambiental. En el estudio realizado prevalecen los enfoques socioecológico y crítico de la educación ambiental. Estas prácticas se vinculan a la construcción de conocimentos aplicables a la solución de los problemas ambientales, aunque aún no reconocen las implicaciones políticas de la dimensión social del medio ambiente, por lo que predominan en la práctica las referencias al sujeto individual sobre el sujeto social o colectivo.

\section{Referencias}

ABRIC, Jean. Coopération, compétition et représentations sociales. Cousset: Del Val, 1987.

ABRIC, Jean. Prácticas sociales y representaciones. México, DC: Coyoacán, 2001.

ALLEN, Lauren; CROWLEY, Kevin. Moving beyond Scientific Knowledge: leveraging participation, relevance, and interconnectedness for climate education. International Journal of Global Warming, Suiza, v. 12, n. 3-4, p. 299-312, 2017. 
ALVES, Vilmar et al. Una mirada a la educación ambiental y movimientos populares ambientales en América Latina. Revista Eletrônica do Mestrado em Educação Ambiental, Rio Grande, ed. esp. p. 6-35, 2017. XVI Encontro Paranaense de Educação Ambiental.

BARENDREGT, Bart; RIVKE, Jaffe (ed.) Green consumption: the global rise of eco-chic. New York: Bloomsbury, 2014.

CALIXTO-FLORES, Raúl. Investigaciones y prácticas pedagógicas en educación ambiental. México, DC: IPEP, 2018.

CALIXTO-FLORES, Raúl; MORENO, Leticia. Educación ambiental en las instituciones de educación superior. México, DC: ReDIE, 2017.

CASTRO, Ricardo de. Educación ambiental. In: ARAGONÉS, Juan; AMÉRIGO, María. Psicología ambiental. Madrid: Pirámide, 2000. p. 357-380.

CRESWELL, John. Qualitative inquiry and research design: choosing among five approaches. London: Sage, 2013.

CRUTZEN, Paul; STOERMER, Eugene. The anthropocene. Global Change Newsletter, Estocolmo, n. 41, p. 17-18, 2000.

CUEVAS, Yazmín. Recomendaciones para el estudio de representaciones sociales en investigación educativa. Cultura y Representaciones Sociales, México, DC, v. 11, n. 21, p. 109-140, 2016.

CUEVAS, Yazmín; MIRELES, Olivia. Representaciones sociales en la investigación educativa. Perfiles Educativos, México, DC, v. 38, n. 153, p. 65-83, 2016.

FREIRE, Paulo. Pedagogía de la esperanza: un encuentro con pedagogía del oprimido. México, DC: Siglo XXI, 1998.

GAD0TTI, Moacir. Ecopedagogía y educación para la sostenibilidad: perspectivas actuales de la educación. México, DC: Siglo XXI, 2003.

GIMENO, José; PÉREZ, Ángel. Comprender y transformar la enseñanza. Madrid: Morata, 1992.

GONZÁLEZ-GAUDIANO, Edgar; ARIAS, Miguel (coord.). La investigación en educación ambiental para la sustentabilidad en México 2002-2011. México, DC: Anuies-Comie, 2015.

GONZÁLEZ-GAUDIANO, Édgar; MEIRA, Pablo Ángel. Educación para el cambio climático. Perfiles Educativos, México, DC, v. 42, n. 168, p. 157-174, 2020.

HART, Paul. Environmental education. In: ABELL, Sandra; LEDERMAN, Norman (ed.). Handbook of research on science education. Mahwah: Lawrence Erlbaum, 2007. p. 689-726.

HEIMLICH, Joe; ARDOIN, Nicole. Understanding behavior to understand behavior change: a literature review. Environmental Education Research, London, v. 14, n. 3, p. 215-237, 2008. 
HURSHA, David; HENDERSON, Joseph; GREENWOOD, David. Environmental education in a neoliberal climate. Environmental Education Research, London, v. 21, n. 3, p. 299-318, 2015.

JACOBI, Pedro. Educação ambiental: o desafio da construção de um pensamento crítico, complexo e reflexivo. Educação e Pesquisa, São Paulo, v. 31, n. 2, p. 233-250, 2005.

JODELET, Denise. La representación social: fenómenos, conceptos y teoría. In: MOSCOVICI, Serge. Psicología social II: pensamiento y vida social. Barcelona: Paidós, 1986. p. 469-493.

KRASNY, Marianne; DILLON, Justin (ed.). Trading zones in environmental education: creating transdisciplinary dialogue. New York: Peter Lang, 2012.

KYBURZ-GRABER, Regula. Socioecological approaches to environmental education and research: a paradigmatic response to behavioral change orientations. In: STEVENSON, Robert et al. (ed.). International handbook of research on environmental education. New York: Routledge, 2013. p. 324-341.

LOUV, Richard. The nature principle: human restoration and the end of nature-deficit disorder. Chapel Hill: Algonquin Books, 2011.

MEIRA, Pablo Ángel. Educación ambiental en tiempos de catástrofe: la respuesta educativa al naufragio del prestige. Educação e Pesquisa, São Paulo, v. 31, n. 2, p. 265-283, 2005.

MEIRA, Pablo Ángel. Problemas ambientales globales y educación ambiental. Integra Educativa, La Paz, v. 6, n. 3, p. 29-64, 2013.

MONROE, Martha et al. Identifying effective climate change education strategies: a systematic review of the research. Environmental Education Research, London, v. 25, n. 6. p. 791-812, 2017.

MORERA, Jaime et al. Theoretical and methodological aspects of social representations. Texto \& Contexto, Florianópolis, v. 24, n. 4, p. 1157-1165, 2015.

MOSCOVICI, Serge. El psicoanálisis, su imagen y su público. Buenos Aires: Huemal, 1979.

MOSCOVICI, Serge; HEWSTONE, Miles. De la ciencia al sentido común. In: MOSCOVICI, Serge. Psicología social II: pensamiento y vida social. Barcelona: Paidós, 1986. p. 679-710.

NIEVES, Mayra. Actores en movimiento(s): prácticas sociales, movilización comunitaria y procesos de cambio en el mundo rural. Revista Textual, México, DC, n. 63, p. 63-81, 2014.

O'DONOGHUE, Rob; RUSSO, Vladimir. Emerging patterns of abstraction in environmental education: a review of materials, methods and professional development perspectives. Environmental Education Research, London, v. 10, n. 3, p. 331-351, 2004.

PAIGE, Kathryn; LLOYD, David; CHARTRES, Mike. Moving towards transdisciplinarity: an ecological sustainable focus for science and mathematics pre-service education in the primary/middle years. AsiaPacific Journal of Teacher Education, Reino Unido, n. 36, p. 19-33, 2008. 
PALMER, Joy. Life experiencies of environmental educators: first report on autobiographical research data: environmental education. London: Routledge, 1992.

PAVLOVA, Margarita. Design and technology education for sustainable future. In: STABLES, Kay; KEIRL, Steve (ed.). Environment, ethics and cultures. Rotterdam: Sense, 2015. p. 87-99.

PAYNE, Phillip. "Amnesia of the moment" in environmental education. The Journal of Environmental Education, United Kingdom, v. 51, n. 2, p. 113-143, 2020.

PIASENTIN, Flora; ROBERTS, Lin. What elements in a sustainability course contribute to paradigm change and action competence? A study at Lincoln University, New Zealand. Environmental Education Research, London, v. 24, n. 5, p. 694-715, 2018.

REIGOTA, Marcos. Meio ambiente e representação social. São Paulo: Cortez, 1995.

REYES, Javier. La educación popular y la dimensión ambiental del desarrollo. documento de discusión. Santiago de Chile: Ceaal, 1984.

ROUQUETTE, Michel Louis. Representations et pratiques sociales: une analyse théorique. In: GARNIER, Catherine; ROUQUETTE, Michel (ed.). Representations sociales et éducation. Montréal: Nouvelles, 2000. p 133-142.

SANDERS, Dawn; RYKEN, Amy; STEWART, Katherine. Navigating nature, culture and education in contemporary botanic gardens. Environmental Education Research, London, v. 24, n. 8, p. 1077-1084, 2018.

SAUVÉ, Lucié. Au coeur des questions socio-écologiques: des savoirs à construire, des compétences à développer. Éducation Relative à L'enrivonnement, Montreal, n. 11, p. 19-40, 2013.

SAUVÉ, Lucié. Educación ambiental y ecociudadanía: un proyecto ontogénico y político. Revista Eletrônica do Mestrado em Educação Ambiental, Rio Grande, ed. esp. p. 261-278, 2017. XVI Encontro Paranaense de Educação Ambiental.

SCHÖN, Donald. El profesional reflexivo: cómo piensan los profesionales cuando actúan. Barcelona: Paidós, 1998.

SCOTT, William. Education for Sustainable Development (ESD): a critical review of concept, potential and risk. In: JUCKER, Rolf; MATHAR, Reiner (ed.). Schooling for sustainable development in Europe. Dordrecht: Springer, 2015. p. 47-70.

STEVENSON, Robert. Schooling and environmental education: contradictions in purpose and practice. Environmental Education Research, London, v. 13, n. 2, p. 139-153, 2007.

TUCK, Eve; MCKENZIE, Marcia; MCCOY, Kate. Land education: Indigenous, post-colonial, and decolonizing perspectives on place and environmental education research. Environmental Education Research, London, v. 20, n. 1, p. 1-23, 2014. 
WWF. World Wildlife Fund. Living planet report 2012: biodiversity, biocapacity and better choices. Switzerland: WWF, 2012.

Recibido en: 05.03.2020

Revisado en: 02.06.2020

Aprobado en: 30.06.2020

Raúl Calixto-Flores es profesor investigador de la Universidad Pedagógica Nacional, México. 